International Journal of Automotive and Mechanical Engineering (IJAME)

ISSN: 2229-8649 (Print); ISSN: 2180-1606 (Online);

Volume 13, Issue 2 pp. 3293 - 3308, September 2016

CUniversiti Malaysia Pahang Publishing

DOI: https://doi.org/10.15282/ijame.13.2.2016.2.0274

\title{
Resonance mode detuning in rotor systems employing active and passive magnetic bearings with controlled stiffness
}

\author{
G. Martynenko \\ Department of Dynamics and Strength of Machines, \\ National Technical University "Kharkiv Polytechnic Institute", \\ NTU “KhPI”, 21, Frunze str., 61002, Kharkiv, Ukraine \\ E-mail: gmartynenko@ukr.net \\ Phone: +380577076343
}

\begin{abstract}
In the paper, the investigator offered a method of reducing the amplitude of vibrations of turbo machinery rotors with passive and active magnetic bearings in resonances and resonance zones corresponding to one of the critical speeds ranging from zero to operating rotational ones. The method was based on the ability to vary the nonlinear force characteristic and the damping properties of active magnetic bearings and passive magnetic bearings of a novel design by changing the electric parameters of electromagnet circuits. The offered design of a passive magnetic bearing with permanent ring magnets and a control winding enabled a short-time change of rotor bearing stiffness. Such rotor bearings can realise the method of detuning from critical speeds during rotor speed-up and run-down. Earlier, this method was feasible only when active magnetic bearings were used. However, a complete magnetic suspension of a rotor with the employment of two radial passive bearings and one axial active magnetic bearing was advantageous as compared to three active magnetic bearings. The feasibility of the detuning method had been substantiated by study results. The study presented the results of numerical analysis, which simulated the process of transition through resonances of the initial system with a significant reduction of vibration amplitudes. The nonlinear system of differential magnetic-mechanical equations was solved using the 5th-order Runge-Kutta method with a validation for the duality of solutions. The results were shown as 3-dimensional spectra of displacement of bearing points and amplitude-frequency responses. These results had been confirmed by experimental data obtained for a laboratory setup that implemented a combined passive-active magnetic suspension of a rotor. The overall total relative error of measurements was maintained at less than $0.5 \%$. The discrepancy between the experimental and design data for amplitudes was within 2-3\%, and for resonance frequencies, it was less than $0.2 \%$. Hence, these studies substantiated the feasibility of passive magnetic bearings with controlled stiffness. They also confirmed the possibility of using passive-active magnetic suspensions for lightweight rotors (e.g., expanders and compressors) with the implementation of the suggested method of detuning from resonance modes.
\end{abstract}

Keywords: passive and active magnetic bearings, rotor dynamics, resonance mode detuning, stiffness-controlled system

\section{INTRODUCTION}

One of the methods of reducing vibrations in rotor machines in different applications is 
to use the self-centering rotor effect, which is manifested during a supercritical operation [1]. The model in Figure 1 illustrates the physical essence of this phenomenon. It implies that when an unbalanced rotor reaches the critical rotational speed $\omega$, the direction of deflection $y$ and eccentricity $e$ become opposite, the centre of mass $C$ is displaced and occupies a position between the geometric centre $O$ and the axis of rotation $O^{*} z$ (Figure 1(a)). Further increasing of the rotational speed makes the absolute value of shaft deflection approach to the eccentricity $e$ (Figure 1(b)), i.e. the centre of mass approaches the axis of rotation. For rotors in rigid supports, the value of the first critical velocity is comparatively big. In this case, the zone of operating rotational speeds can be located at an insufficient distance (for reducing rotor vibration amplitudes) from the critical speed one (Figure 1(b)). In practice, one of two methods is used for rotor machine resonance mode detuning. The first method implies displacement of increased vibration zones in the increasing direction so that the entire range of operating rotational speeds is located in advance of the first critical speed (rigid rotor). The second method implies machine operation in supercritical zones (a flexible rotor)[2]. In this case, it is necessary to reduce the first critical rotational speed, i.e. the increased vibration zone threshold, and at the same time to ensure safe transition through critical speeds in the range of up to the maximal operating rotational ones $[2,3]$. This is achieved by reducing the stiffness of the "rotor-support" dynamic system. In order to do this, the rotors are mounted in elastic supports (ES) with specified stiffness. The vibration amplitudes can be reduced with the help of elastic-dampening supports (EDS) by introducing artificial dampeners into their design. This decreases the amplitudes of resonance vibrations; however, machine efficiency will drop.

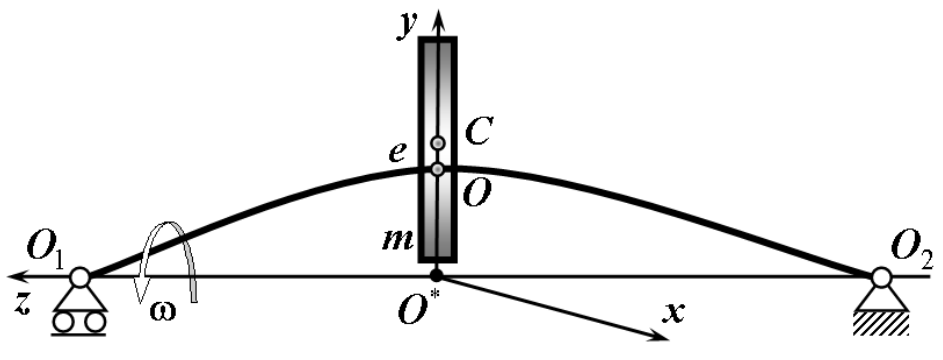

(a)

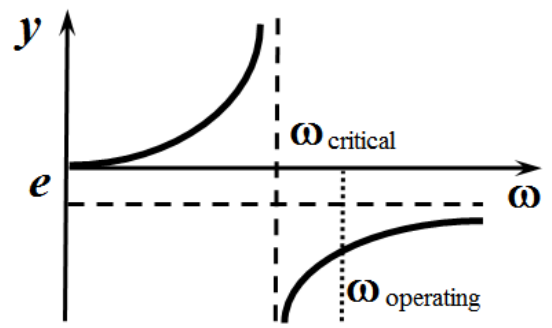

(b)

Figure 1. (a) Rotor model; (b) Shaft deflection vs. rotational frequency.

Figure 2 shows rotor vibration modes in different supports corresponding to the first three critical rotational speeds. For extremely yielding supports, the rotor passes the first two critical speeds with the formation of cylindrical and conical precession (Figure 2(a)). A curved vibration mode corresponds to the third critical speed. With rigid supports, all rotor vibration modes corresponding to the first three critical speeds are curved (Figure 2(c)). When support rigidity is commensurable with shaft stiffness (Figure 2(b)), the vibration modes are a combination of modes for these two cases. The most common vibrations of rotors are caused by static and moment imbalance [3]. The amplitudes of such vibrations can be reduced dramatically by utilising the properties of a stiff rotor in an ES. Figure 3 shows several types of such ES. However, there are also other kinds of nuisance vibrations directly related to using mechanical ES. They are autovibrations caused by the properties of the oil film in sleeve bearings (Figure 3(a)); different kinds of nonlinear vibrations related to nonlinearity of the force characteristics of roller bearings 
(Figure 3(b)) as preloaded systems, and others.

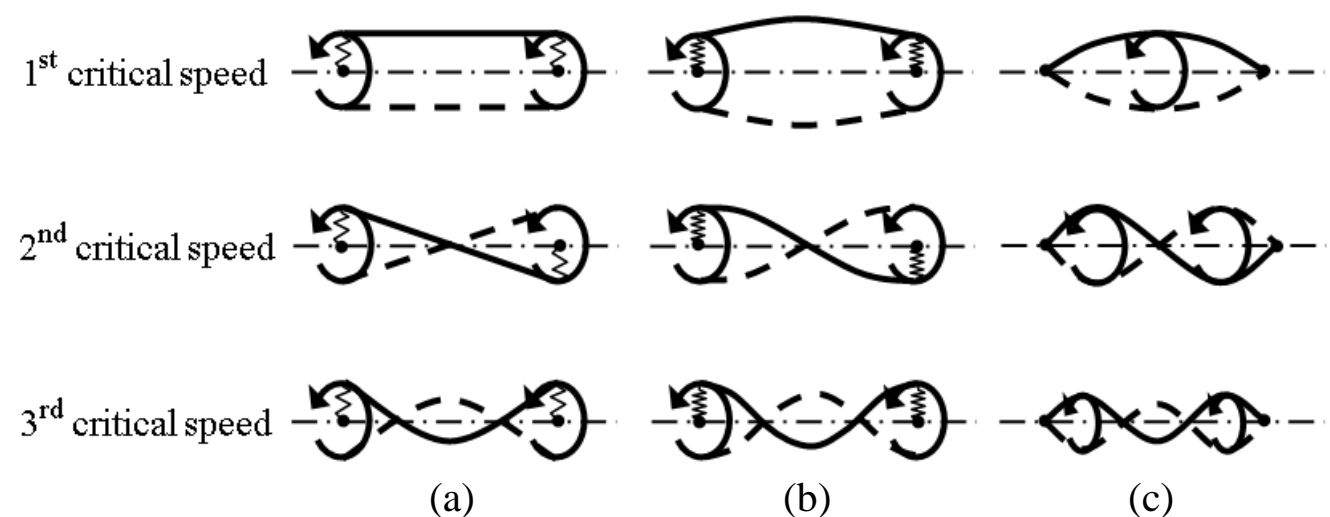

Figure 2. Lateral vibration modes of rotors in different supports:

(a) extremely yielding; (b) yielding; (c) rigid.

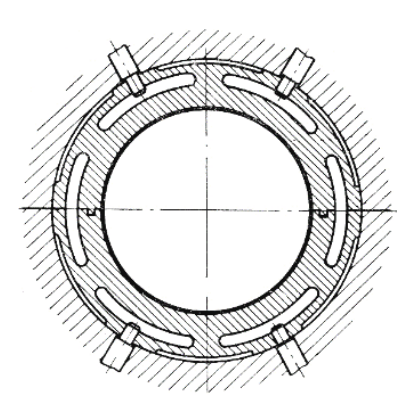

(a)

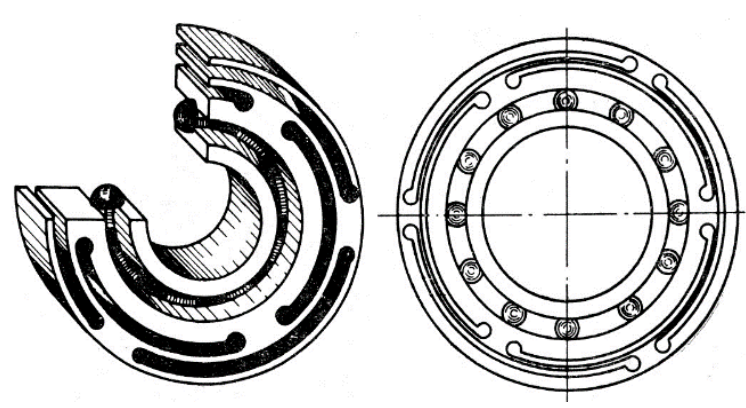

(b)

Figure 3. Elastic rotor supports: (a) sleeve bearing, (b) roller bearing.

If a rotor is mounted in an ES, troubles can occur when a rotor speeds up, passes through critical speeds, and vibrates as a rigid body [2]. This study offered a method of transition through such elevated vibration zones by a short-term ES stiffness change during rotor speed-up or rundown, i.e. stiffness control as a function of rotational speed. Then the ES force characteristics will be adaptive, allowing for the detuning of the "rotor in ES" system from critical speeds over the entire range from zero to operating rotational speeds of the rotor machine. An ES with variable stiffness can be represented not only by conventional active magnetic bearings (AMB) but also by passive magnetic bearings (PMB) with controlled stiffness of the developed design. In an AMB, rotor position stability is ensured by a negative feedback control system (CS) [4-7], whereas in a PMB, this is achieved by self-centering [8]. The force characteristics of these bearings, in contrast to those of mechanical ES, can change by varying the electric parameters (voltages or control currents) [9]. By changing the values of active resistances in AMB circuits, one can control damping by increasing it only in the resonance zones. In this case, AMB and PMB will be an EDS with variable parameters. Yet another feature of AMBs to be mentioned and which can have a dramatic effect on the practical applicability of the given detuning method is the nonlinear dependence of their force characteristics on the displacement of rotor support sections (i.e. on the gap between the journal and stator poles) as well as on the currents in the pole coil windings. They change as a function of rotor position defined by the control system, according to a pre-set law. 
Presently, there are several analytical and practical approaches to implementing the resonance detuning method. Thus, Klimnjuk [10] and Ehskin [11] proposed a method of safe speed-up of horizontal and vertical massive rotors of turbomachines installed in active magnetic bearings. It assumed a rapid rotor transition through the critical speeds of the first two rotor modes (modes corresponding to a rotor as a stiff body). This was achieved by using an automatic control system to set up several running conditions with various stiffness in active magnetic bearings. As the rotor gains speed according to a program set by the bearing's automatic control system, one running condition changes smoothly (bumpless) from another one and vice versa so that rotor operation under resonance conditions and in the resonance zone of any running condition from zero to operating speed is eliminated completely during speed-up. Practical implementations of similar methods of resonance detuning and damping excessive vibration amplitudes are also known. Thus, the firm SKF S2M (SKF Group), which specialises in manufacturing $\mathrm{AMB}$, uses anticipatory control of AMB parameters in different turbomachinery employed in the oil-and-gas industry [12]. Patented algorithms (registered trademarks), such as ODC (Optimal Damping Control), ABS (Advanced Balancing System), and AVR (Automatic Vibration Reduction) provide enhanced vibration control and are realised using Control Cabinet E300V2 [13].

A distinctive feature of the described approaches to implementing a method for detuning from resonances and damping elevated oscillation amplitudes is that they are all used in complete magnetic suspensions of rotors only in AMB. However, PMB are known to possess a host of advantages as compared to AMB (e.g., they need no electric power sources and an automatic control system that comprises of the bulk of an AMB cost) [5]. The objective of the study was developing a new kind of passive magnetic bearing with short-time variable stiffness in a range sufficient for realising the method of detuning the rotor from resonance modes during speed-up and run-down. The method consisted of utilising the possibility of rapidly changing the stiffness and/or damping parameters in magnetic bearings of different types (and not only in an AMB) with a control system, including, along with position sensors, an angular speed sensor. Besides that, the objective of the study was also the numerical and experimental validation of the suggested method of detuning the rotor from critical speeds (passing through resonances) during speed-up and run-down. Another objective was proving the feasibility of the method for different rotor machines, such as small and middle-sized expanders and compressors. In addition to known approaches described by [14, 15] using the suggested passive-active magnetic suspension with controlled stiffness will allow for the reduction in cost and increase the effectiveness of different gas turbines, turbo-expanders, and expandercompressor units [16] by excluding friction losses and damping the vibration activity of rotors.

\section{METHODS AND MATERIALS}

\section{Detuning a Rotor in an MB from Critical Rotational Speeds}

A distinctive feature of the described approaches to implementing the method is that they are all used in complete magnetic suspensions of rotors only in AMB. However, PMB are known to have a range of advantages in comparison to AMB (for instance, they need no electric power sources and no automatic control system that contributes to the basic share of AMB cost) [6, 8]. A basic deficiency of PMB is the impossibility to develop a complete rotor magnetic suspension with only the use of permanent magnets due to Earnshaw's theorem [17] (on the instability of the position of equilibrium of an active element in a 
static force field if the force and distance are related by the inverse square law) and Brounbeck's statements [18]Error! Reference source not found. (stable body suspension in a static magnetic field is possible when the magnetic permeability of the body material is less than that of the environment). A generalisation of these provisions carried out in a study [19] allowed for the drawing of a conclusion that under usual atmospheric conditions, only bodies made of diamagnetics or superconductors can be suspended in a static magnetic field. Ferromagnetic bodies can be suspended only in the case where the environment shall be a ferromagnetic fluid with a higher magnetic permeability. To ensure stable positioning of permanent magnets in three directions, study [8] offered submerging them in a ferromagnetic fluid, which can be an oil-based synthetic ether with a magnetisation saturation of $325 \mathrm{Gs}$ and a viscosity of $0.13 \mathrm{~Pa} \cdot \mathrm{s}$. Using magnetic fluids for improving properties is known also for other types of bearings [20]. Hence, complete rotor suspension with PMB for the majority of rotor machinery is infeasible from both the technological and operational viewpoints. Therefore, based on a reasonable balance between advantages and drawbacks, complete magnetic suspension can be implemented in practice, whether by using controlled AMBs as all rotor bearings, or by using combined magnetic suspensions with $\mathrm{PMB}$ and $\mathrm{AMB}$ with various design options. The most practical approach would be to use a combination of magnetic bearings of different types in medium-sized high-speed rotor machinery, e.g., turbo-expanders, expander-generator and expander-compressor units [5]. They can use two radial PMBs and one axial AMB arranged in the centre or at one end of the shaft. This is due to the design features, such as the presence of one or two discs arranged on the rotor cantilevers [16]. This study offered a new type of passive magnetic bearing with a short-term variable stiffness in a range sufficient for implementing the investigated method of rotor resonance detuning during speed-up and rundown. The suggested method of detuning the rotor from critical speeds (transition through resonances) during speed-up or rundown implies that the control system (CS) changes rapidly the stiffness and/or damping parameters of magnetic bearings of different types (and not only those of AMB). Besides having position sensors, the CS also has an angular speed sensor [9].

\section{Implementation with AMB}

The structural diagram of a variant of a control system implementing the method for a radial AMB is shown in Figure 4. AMB stiffness properties can be varied within a certain range of angular speeds by changing the values of the control voltages $u_{c 1}, \ldots, u_{c 4}$. This results in a change of average currents $i_{c 1}, \ldots, i_{c 4}$ (bias currents) in the windings of electromagnets and allows for the changing of the force characteristics of the support [21]. The AMB damping properties can be increased for a short time by increasing the active resistances $r_{c 1}, \ldots, r_{c 4}$ in the circuits of the electromagnets in the zone of rotor critical rotational speeds. This will reduce the amplitudes of resonance vibrations [21].

The algorithm of operation of the control system suggested assumes prior selection of two (or more) operating conditions. In the first condition (the designed one), the AMB force characteristics should ensure the required motion stability of the supercritical rotor in a certain range of angular velocities spanning the operating rotational speeds. The second operating condition assumes operation with greater (lesser) support stiffness as compared to the first case. The stiffness should be such that the first critical speeds of the rotor are higher (lower) as compared to the system operating in the first (designed) condition. Rotor speed-up is initiated in the first operating condition. Then, as the rotor approaches the critical zones, the operating condition is switched to the second one, and when the resonance areas of the first operating condition have been passed, the operating 
condition is switched back to the first (designed) one. If there are several resonances prior to the onset of the operating rotational speeds, successive switching from the first operating condition to the second one and vice versa will exclude completely the vibrations with increased amplitudes. During rotor rundown, the process of transitioning through resonance is similar. In general, several such operating conditions can exist. In this case, switching between them should take place according to a predesigned program.

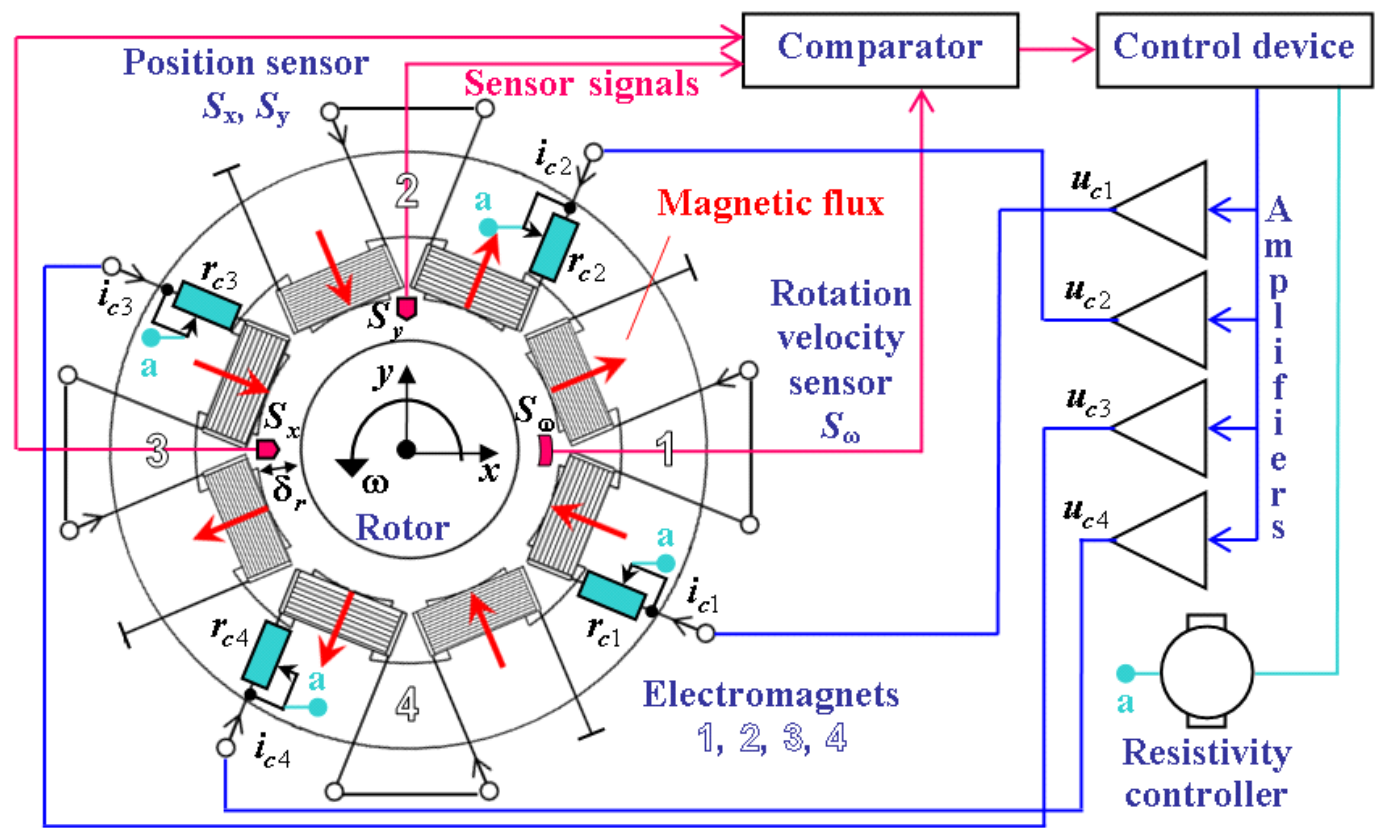

Figure 4. Structural diagram of a CS for controlling rotor motion in a radial AMB with controlled stiffness and damping parameters.

Figure 5 is a schematic presentation of how the supercritical rotor reaches operating rotational speeds and passes through the first resonance (critical speed). The diagram shows the dependence of the amplitude of the first forced vibrations harmonic $\left(A_{1}\right)$ on the frequency $(\omega)$ of the harmonic driving force for a stiff AMB force characteristic. $A_{1}{ }^{\prime}$ and $A_{1}{ }^{\prime \prime}$ designate the resonance curves for different stiffness values, which are ensured by different values of average currents or bias currents. The resonance curve $A_{1}{ }^{\prime \prime}$ corresponds to bigger stiffness values, and curve $A_{1}{ }^{\prime}$ is the amplitude-frequency response (AFR) of the design operating condition. The dashed lines in Figure 5 are skeleton curves $\omega_{0}{ }^{\prime}$ and $\omega_{0}{ }^{\prime \prime}$ corresponding to different stiffness values. Solid lines designate the resulting system AFR obtained when using the method proposed. It is implemented by transition from one resonance curve $A_{1}{ }^{\prime}$ to another one $A_{1}{ }^{\prime \prime}$ by changing the stiffness of magnetic bearings in a pre-set range $\left[\omega_{1} \mathrm{~min}, \omega_{1 \mathrm{max}}\right]$. In this case, the maximum values of vibration amplitudes $A_{1 \text { max }}$ are significantly smaller than the maximums $A_{1}{ }^{\prime}$ max and $A_{1}{ }^{\prime \prime}$ max of both resonance curves $A_{1}{ }^{\prime}$ and $A_{1}{ }^{\prime \prime}$.

The vibration amplitude near the resonance can also be reduced by increasing the damping in the system. This is achieved by increasing the active resistances in the circuits of the coils of the AMB electromagnets (Figure 5, curve $A_{1}{ }^{\prime \prime}$ ) [21]; however, this extends the resonance zone and results in additional energy consumption. 


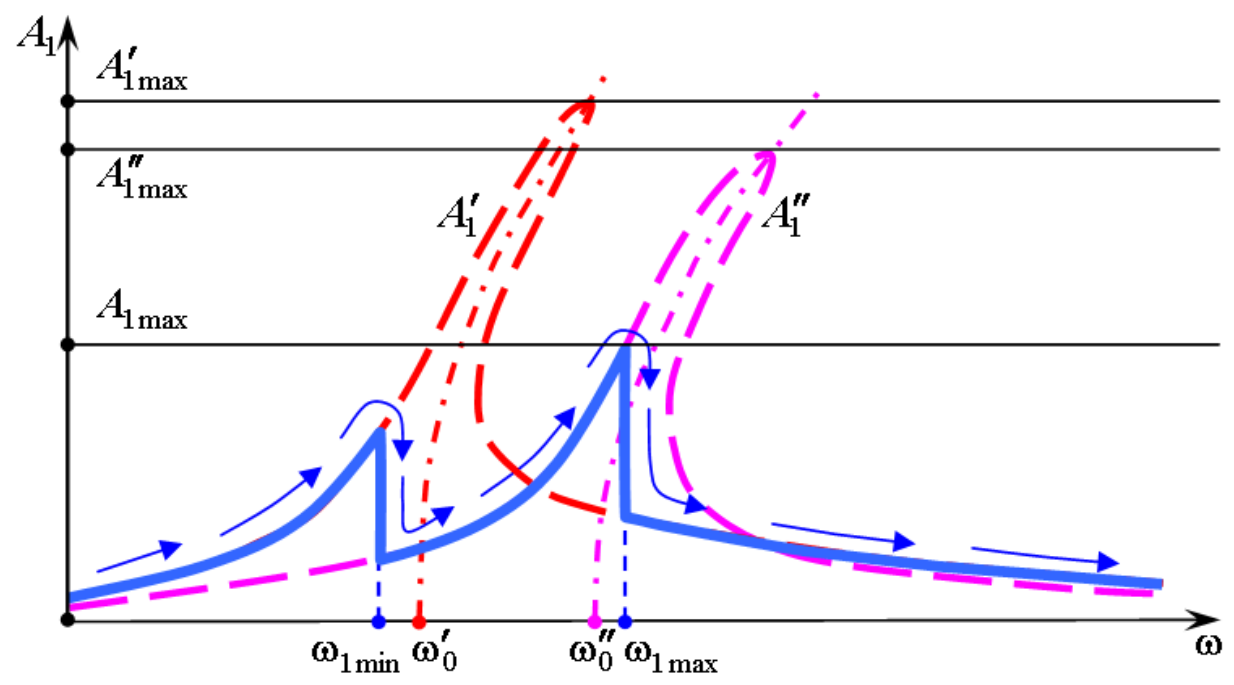

Figure 5. Schematic presentation of the AFR of a rotor in an AMB with controlled stiffness.

\section{Implementation with a PMB}

The most promising design solution for radial PMBs with permanent magnets is using magnetic rings. A big variety of designs of radial passive magnetic bearings with permanent ring magnets are known [8]. They differ first as to the direction of ring magnetising (axial or radial), and second, by the operating principle (repulsion or attraction). A straightforward option for technical implementation is the PMB design with two permanent ring magnets based on repulsion [9, 22]. The results of analytical and experimental studies in stiffness parameters, namely the dependence of ponderomotive forces and quasi-elastic coefficients on the rotor radial position lead to a conclusion that it is preferable to use permanent magnets with axial magnetising in PMB, with all other geometric and physical parameters being equal. A schematic diagram of such a PMB and the pattern of equipotential magnetic file lines are shown in Figure 6. It shows that the radial PMB considered is stable only in the radial direction, and in the axial one, it is unstable. For the given radial PMB, the stiffness characteristics can be changed by creating an additional electromagnetic field of the same configuration as that of the field created by an external fixed permanent ring magnet. In case the force line directions of these magnetic fields match, the magnetic forces and the quasi-elastic coefficients of such a support will increase, and if the force lines are opposite, they will decrease. For the PMB with axial magnetising being considered, such a field can be created with a winding wound onto an external cylindrical surface of a fixed ferromagnetic ring. A model of such a PMB is shown in Figure 6(c).

The principle of action of a PMB with controlled stiffness of the design suggested is as follows. When a d.c. supply voltage is applied to the coil and currents specified above the flow in the circuit, the force and quasi-elastic coefficients change their values as in the previous cases. Thus, when creating a magnetic field that matches the field induced by the fixed permanent ring magnet (main one), the restoring force can change up to $36 \%$, and the stiffness coefficient can change up to $50 \%$. If the flowing current shall create a magnetic field in opposition to the main one, the parameters considered will decrease by roughly the same values. Identical principles are used, for example, in the design of a Magneto-Rheological Fluid Damper [23]. 


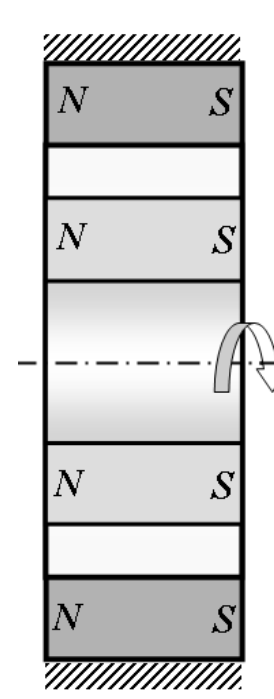

(a)

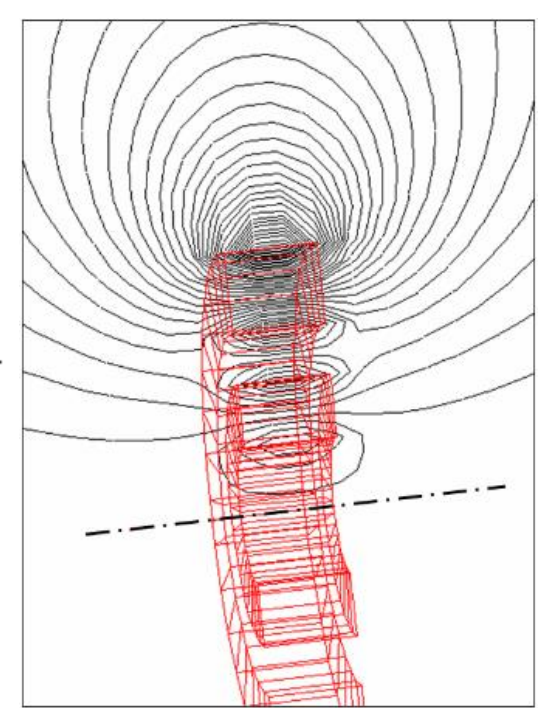

(b)

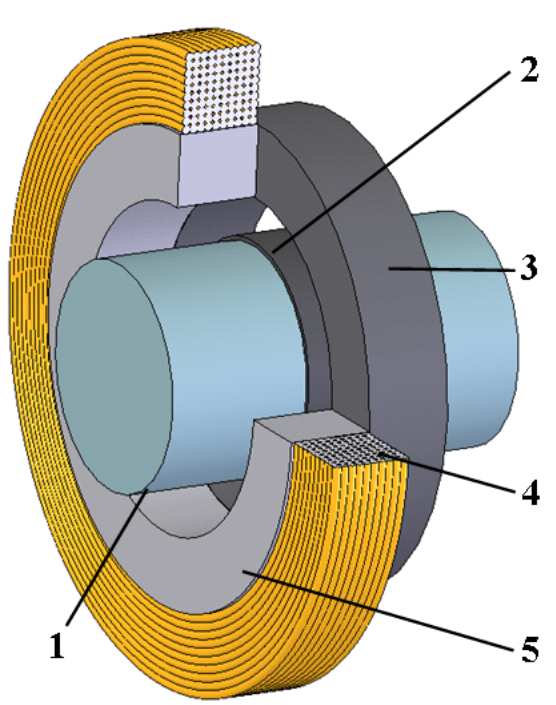

(c)

Figure 6. Radial magnetic bearing with two permanent ring magnets: (a) schematic diagram; (b) equipotential magnetic field lines; (c) solid body model of a PMB with controlled stiffness: 1 - rotor, 2 - permanent ring magnet of the rotor, 3 - permanent ring magnet of the stator, 4 - control winding, and 5 - ferromagnetic annular core.

Note that when the coil is arranged, only on one side an axial expulsive force originates and acts in the direction of the axis of cylinders onto the movable permanent magnet away from the coil (Figure 6(c)). This can be accounted for in this case by the non-symmetry of the magnetic field with respect to the plane of the perpendicular axis of magnets passing through their centres of gravity. This magnetic field's non-symmetry can be eliminated either by introducing the magnetic bearing design of a second coil on the other side of the magnetic stator ring or by mirror positioning the two radial PMB. Next, this PMB is introduced as an actuator into the control system identical to that used in an AMB (Figure 4) with the sole difference that it has only an angular speed sensor. Using the supports suggested in case of a super-resonance rotor when it reaches the rated speed, this will allow the transition through critical speeds corresponding to rotor vibrations as a solid body without significant vibration amplitude increases. Thus, when the angular speed reaches a value close to the critical one, detuning from specified resonance modes can be achieved by the short-term actuation of electromagnets (applying d.c. voltage to them) and by increasing or reducing the stiffness of supports. After these modes have been passed, voltage from electromagnets is removed and the stiffness of supports is defined further by characteristics corresponding to zero current in the bearing winding. Such PMB with controlled stiffness can be used in small and medium-sized rotor machinery with operating speeds within the critical speeds of translational and deformation modes (motions of the conical/cylindrical precession and cambered axis precession type).

\section{RESULTS AND DISCUSSION}

\section{Laboratory Model of a Rotor in an MB for Testing the Method}

To validate the functionality of the method suggested, a series of numerical experiments for a model of a magnetic suspension of a small-size high-speed rotor were conducted. A laboratory setup of a rotor in a complete combined passive-active magnetic suspension 
and a PMB with controlled stiffness are shown in Figure 7. The support was made effective by two radial PMB and one axial AMB. Such scheme of rotor suspension can be suggested, for example, for the application in rotors of expansion-compression units.

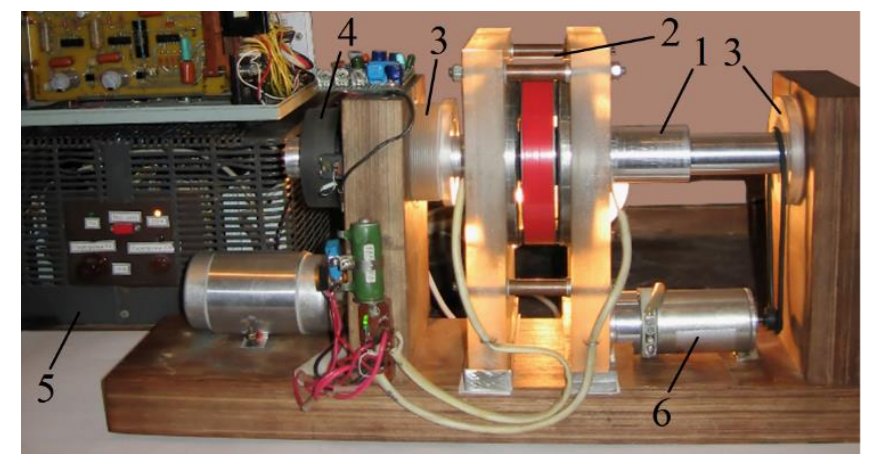

(a)

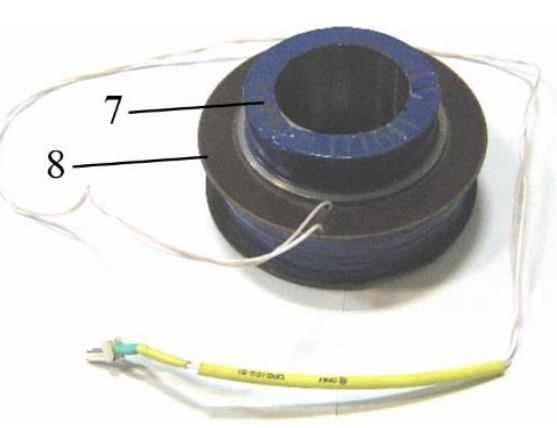

(b)

Figure 7. (a) Laboratory setup; (b) PMB with controlled stiffness: 1 - rotor, 2 - axial double-action AMB with two electromagnets, 3 - radial PMB with controlled stiffness, 4 - axial position encoder, 5 - axial AMB CS units, 6 - electric motor with shaft drive,

7 - permanent ring magnet, 8 - control winding on a steel annular core

\section{Mathematical Model of the Dynamics of a Rigid Rotor in Magnetic Bearings}

Figure 8 visualises the design model of a combined magnetic suspension, where $O^{*} x y z$ are fixed right-hand Cartesian coordinates; $O_{1}\left(x_{1}, y_{1}, z_{1}\right), O_{2}\left(x_{2}, y_{2}, z_{2}\right)$ are centres of support sections of the rotor in the radial PMB in permanent ring magnets located at distances $l_{1}, l_{2}$ from the centre of mass $C ; O_{3}\left(x_{3}, y_{3}, z_{3}\right)$ is the centre of the rotor support section in the axial AMB (disk centre of mass); $u_{c 1}, u_{c 2}$ are control voltages applied across the windings of the axial AMB; $i_{c 1}, i_{c 2}$ are currents in the windings of the axial AMB; $e, \gamma$ are linear and angular eccentricities; and $\omega$ is the rotor angular speed. This combined magnetic suspension used magnetic bearings as shown in Figure 9. The radial supports here are self-aligning PMB in a mirrored position to compensate for the axial force introduced by them. Their additional bias winding served for implementing stiffness control. The axial support was a double-side action AMB. Figure 9(a) shows the radial magnetic forces in PMB vs. rotor radial displacement at different values of parameters: $F_{M 0}$ at $i_{b 1,2}=0, F_{M 1}$ at $i_{b 1,2}= \pm 10 \mathrm{~A}(w=150), F_{M 2}$ at $i_{b 1,2}= \pm 3.5 \mathrm{~A}(w=500)$ and $F_{M 3}$ at $i_{b 1,2}= \pm 1.3 \mathrm{~A}(w=1,500)$, where $w$ is the number of turns in windings [9]. Figure 9(b) shows the axial magnetic forces vs. axial rotor displacement at different maximum voltage values $U_{0}$ applied across AMB windings according to the pre-set control algorithm $u_{c 1}\left(z_{3}\right), u_{c 2}\left(z_{3}\right)$ [9]. These force characteristics had been proven experimentally [9].

The dynamics of a rigid rotor in a combined magnetic suspension is described mathematically by a system of five differential equations, nonlinear with respect to the generalised coordinates $\mathbf{q}=\left\{x_{1}, y_{1}, x_{2}, y_{2}, z_{3}\right\}$ and their time derivatives [24]:

$$
\left\{\begin{array}{l}
m_{11} \ddot{x}_{1}+m_{12} \ddot{x}_{2}+m_{13} \omega\left(\dot{y}_{1}-\dot{y}_{2}\right)+b_{x 1} \dot{x}_{1}+f_{x 1}^{\prime \prime}+f_{x 1}^{\prime \prime \prime}=F_{M x}\left(x_{1}, y_{1}\right)+Q_{x 1}+H_{x 1}(t), \\
m_{22} \ddot{x}_{2}+m_{12} \ddot{x}_{1}-m_{13} \omega\left(\dot{y}_{1}-\dot{y}_{2}\right)+b_{x 2} \dot{x}_{2}+f_{x 2}^{\prime \prime}+f_{x 2}^{\prime \prime \prime}=F_{M y}\left(x_{1}, y_{1}\right)+Q_{x 2}+H_{x 2}(t), \\
m_{11} \ddot{y}_{1}+m_{12} \ddot{y}_{2}-m_{13} \omega\left(\dot{x}_{1}-\dot{x}_{2}\right)+b_{y 1} \dot{y}_{1}+f_{y 1}^{\prime \prime}+f_{y 1}^{\prime \prime \prime}=F_{M x}\left(x_{2}, y_{2}\right)+Q_{y 1}+H_{y 1}(t), \\
m_{22} \ddot{y}_{2}+m_{12} \ddot{y}_{1}+m_{13} \omega\left(\dot{x}_{1}-\dot{x}_{2}\right)+b_{y 2} \dot{y}_{2}+f_{y 2}^{\prime \prime}+f_{y 2}^{\prime \prime \prime}=F_{M y}\left(x_{2}, y_{2}\right)+Q_{y 2}+H_{y 2}(t), \\
m \ddot{z}_{3}+b_{z 3} \dot{z}_{3}+f_{z 3}^{\prime \prime}+f_{z 3}^{\prime \prime \prime}=F_{M z}\left(z_{3}\right)+Q_{z_{3}}+H_{z_{3}}(t) ;
\end{array}\right.
$$


where $m_{i j}$ are inertia and gyroscopic coefficients including the rotor mass $m$ and the moments of inertia (equatorial $J_{1}$ and polar $J_{3}$ ones) are equal to

$$
m_{11}=\frac{m l_{2}^{2}+J_{1}}{l^{2}} ; m_{12}=\frac{m l_{1} l_{2}-J_{1}}{l^{2}} ; m_{22}=\frac{m l_{1}^{2}+J_{1}}{l^{2}} ; j=\frac{\omega J_{3}}{l^{2}},
$$

$b_{x 1, \ldots, z 3}$ are viscosity coefficients; $f_{q r}^{\prime \prime}\left(x_{1}, \ldots, \ddot{z}_{3}\right)$ and $f_{q r}^{\prime \prime \prime}\left(x_{1}, \ldots, \ddot{z}_{3}\right)$ are nonlinear terms of inertia and potential field forces whose order with regard to generalised coordinates and their derivatives are comparable with that of magnetic forces dependencies (Figure 9) $F_{M y}(x, y), F_{M x}(x, y), F_{M z}(x, y, z) ; Q_{x 1, \ldots, z 3}$ are other generalised forces, in particular, the force of gravity; $H_{x 1, \ldots, z 3}(t)$ are external time-dependent disturbing forces caused, in particular, by nonequilibrium, where

$$
\left\{\begin{array}{l}
H_{x 1}(t)=m_{11} \mathrm{E}_{x}+j \Gamma_{x} ; H_{x 2}(t)=m_{22} \mathrm{E}_{x}-j \Gamma_{x} ; \\
H_{y 1}(t)=m_{11} \mathrm{E}_{y}-j \Gamma_{y} ; H_{y 2}(t)=m_{22} \mathrm{E}_{y}+j \Gamma_{y} ; \\
\mathrm{E}_{x}=e_{1} \cos (\omega t)-e_{2} \sin (\omega t) ; \mathrm{E}_{y}=e_{1} \sin (\omega t)+e_{2} \cos (\omega t) ; \\
\Gamma_{x}=\gamma_{1} \sin (\omega t)+\gamma_{2} \cos (\omega t) ; \Gamma_{y}=\gamma_{1} \cos (\omega t)-\gamma_{2} \sin (\omega t) .
\end{array}\right.
$$

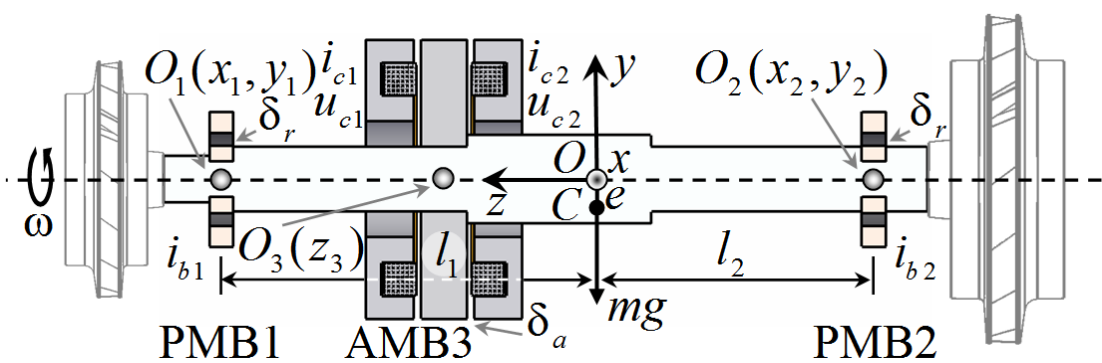

Figure 8. Design model of a rotor in an AMB and PMB with controlled stiffness.

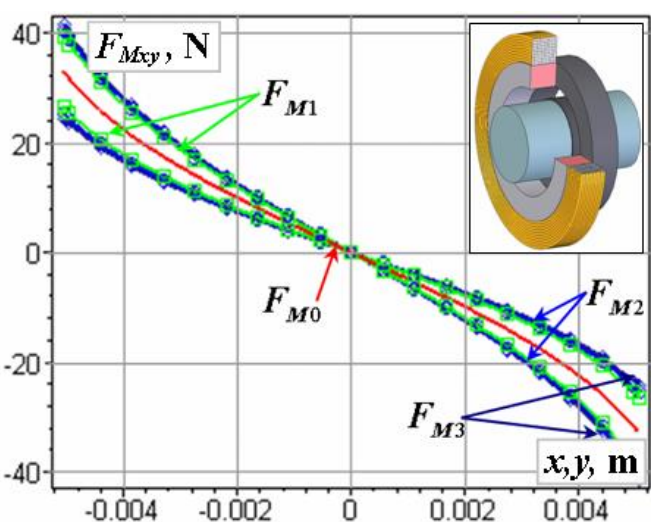

(a)

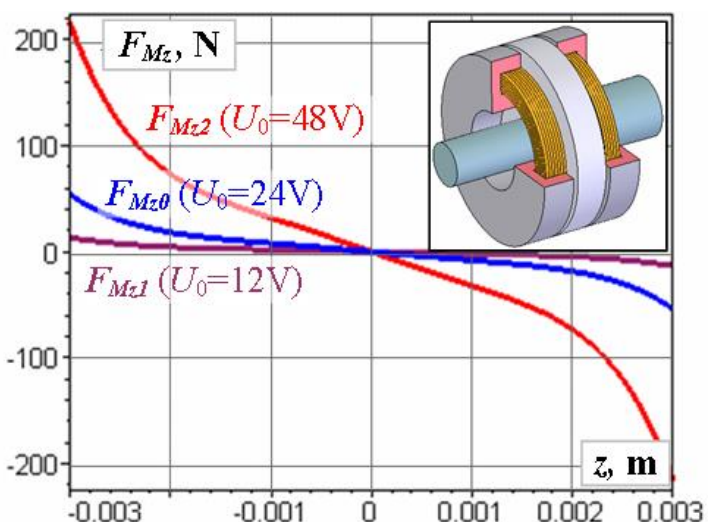

(b)

Figure 9. Forces vs. displacements of rotor support sections in an experimental model: (a) radial PMB with permanent magnets and an additional bias winding; (b) doublesided action AMB.

For the second-order nonlinear terms $f_{q r}^{\prime \prime}\left(x_{1}, \ldots, \ddot{z}_{3}\right)$, it is required to account for the nonlinear features of the electromechanical system being considered, thus the following relations hold: 


$$
\left\{\begin{array}{c}
f_{x 1}^{\prime \prime}=-m \ddot{z}_{3} \mathrm{E}_{x} / l ; f_{x 2}^{\prime \prime}=m \ddot{z}_{3} \mathrm{E}_{x} / l ; f_{y 1}^{\prime \prime}=-m \ddot{z}_{3} \mathrm{E}_{y} / l ; f_{y 2}^{\prime \prime}=m \ddot{z}_{3} \mathrm{E}_{y} / l ; \\
f_{z 3}^{\prime \prime}=m\left(\left[\left(\ddot{x}_{2}-\ddot{x}_{1}\right)-\omega^{2}\left(x_{2}-x_{1}\right)+2 \omega\left(\dot{y}_{2}-\dot{y}_{1}\right)\right] \mathrm{E}_{x}+\right. \\
\left.+\left[\left(\ddot{y}_{2}-\ddot{y}_{1}\right)-\omega^{2}\left(y_{2}-y_{1}\right)-2 \omega\left(\dot{x}_{2}-\dot{x}_{1}\right)\right] \mathrm{E}_{y}\right) / l
\end{array}\right.
$$

The third-order nonlinear terms $f_{q r}^{\prime \prime \prime}\left(x_{1}, \ldots, \ddot{z}_{3}\right)$, are not shown here due to their cumbersome notation; however, it was them that demonstrated the full co-relation between all generalised coordinates with the help of terms having no dependence on unbalance parameters.

\section{Vibrograms of Rotor Vibrations for Different Rotational Speeds}

During numerical modelling, the system of equations (1) was solved with the 5th-order Runge-Kutta method for discrete angular speed values. The many-valuedness of the solution was checked and excluded by multiple computations for each frequency and different initial conditions. In doing so, stationary areas were searched for, whereas time intervals corresponding to transient processes were excluded from consideration. Hence, the results of the numerical analysis of forced vibrations were the solutions for stationary areas and generalised coordinates $x_{1}, y_{1}, x_{2}$, and $y_{2}$ in the angular speed range of 0 $40 \pi \mathrm{rad} / \mathrm{s}$ with a step of $\pi / 2 \mathrm{rad} / \mathrm{s}$. Computational studies were conducted for a laboratory model of a combined magnetic suspension of a rotor with the following parameters (Figure 7) [24]: $m=2.5 \mathrm{~kg}, l_{1}=0.10675 \mathrm{~m}, l_{2}=0.17675 \mathrm{~m}, J_{1}=0.0107386 \mathrm{~kg} \cdot \mathrm{m}^{2}$, $J_{3}=0.003377 \mathrm{~kg} \cdot \mathrm{m}^{2}, \delta_{r}=0.0055 \mathrm{~m}, \delta_{a}=0.003 \mathrm{~m}, b_{x 1, \ldots, y 2}=2.325 \mathrm{~kg} / \mathrm{s}, e=0.00006 \mathrm{~m}$, $\gamma=0.003 \mathrm{rad}$, and dependencies of forces $F_{M 0}$ and $F_{M z 0}$ (Figure 9 The Runge-Kutta fifthorder method was used for numerically solving the system of motion equations (1) for angular speed values in the range of $0-40 \pi$ with a step of $\pi / 2 \mathrm{rad} / \mathrm{s}$. For each angular speed value, the stationary solution was analysed spectrally using the Fast Fourier Transform (FFT).

Figure 10 visualises the results of such order analysis in the form of spectrograms of the generalised coordinates, where $f$ is the spectrum frequency, $\omega$ is the angular speed (rotational speed), and $A$ is the amplitude of the respective generalised coordinate. These are the results of calculating the parameters of forced vibrations of a rotor due to dynamic imbalance. The spectra exhibited both superharmonics and subharmonics. This conformed to the experimental results of [21] and is typical for rotor systems with nonlinear bearings [25]. Besides visualising the results of order analysis, the graphs show vibrograms corresponding to the first resonance mode and the end of the calculation range. It was found that the rotor motion in the first critical speed zone was of the direct cylindrical precession type (Figure 2a). Here, the resonances during vibration in the $x$ and $y$ directions were displaced as per the angular speed by $9.5 \mathrm{rad} / \mathrm{s}$. Besides, these vibrations differed by the presence of superharmonics (Figure 10). At the end of the angular speed calculation range, there was a transition to the second resonance with the occurrence of subharmonics, and the rotor motion corresponded to a direct conical precession (Figure 2a). All these phenomena were in agreement with the experimental studies [24]. This confirmed the adequacy of the mathematical model and the validity of numerical analysis results. Comparative analysis of numerical and experimental results had shown the identity for both qualitative representation of the processes in the system and quantitative determination of their parameters: for the amplitude, the difference was within $2-3 \%$, and for the values of resonant frequencies, the difference was within 0.2 to 
$0.5 \%$ [26]. The adequacy and applicability of mathematical model (1) and the algorithm of its numerical analysis for studying the nonlinear dynamics of rigid rotors in MB [27] of various types had been confirmed experimentally. This was done by comparing not only the AFR over the entire investigated range of excitation frequencies, but also by the dependencies of amplitudes of other harmonics on frequency, as well as by vibrograms, spectra and trajectories of the motion of rotor bearing points [21, 24]. Data were recorded and processed with an instrumentation-and-analysis software-hardware complex built around an analog-digital converter module type ADA-1406 [26]. Data were processed using sequential analysis and the Fast-Fourier Transform method [28]. The overall total relative error of measurements was maintained at less than $0.5 \%$. This allowed for the use this mathematical model for computational modeling of the feasibility of the suggested method for detuning rotors from resonance modes and reducing vibration amplitudes.

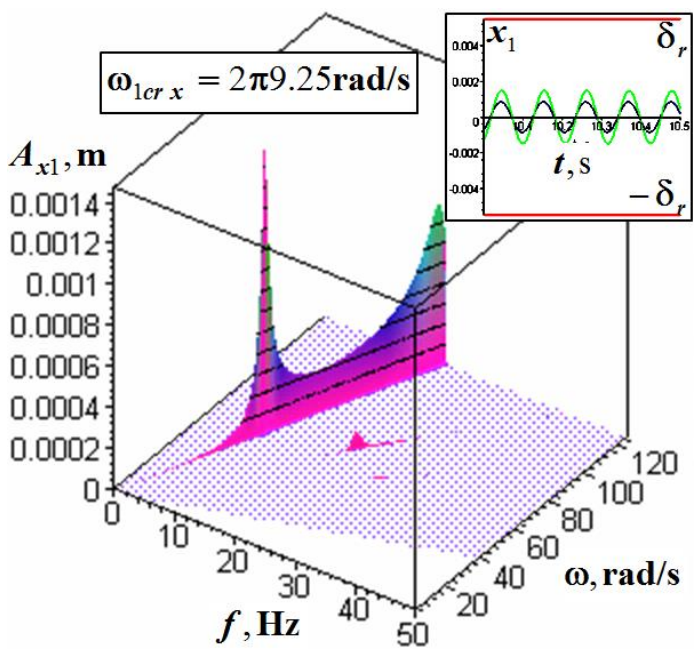

(a)

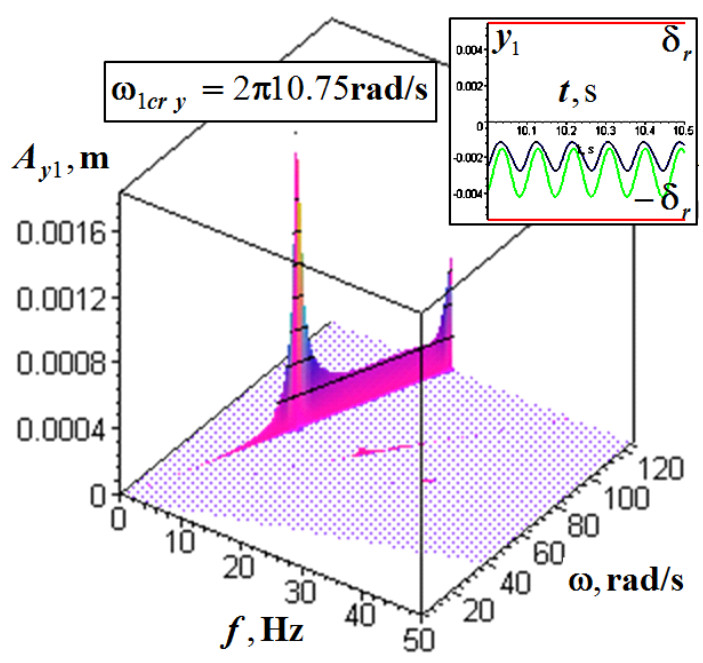

(c)

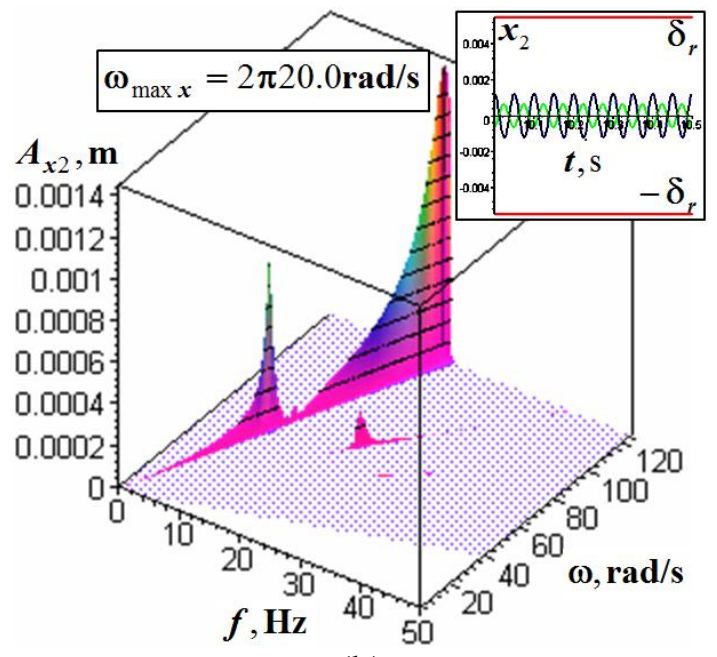

(b)

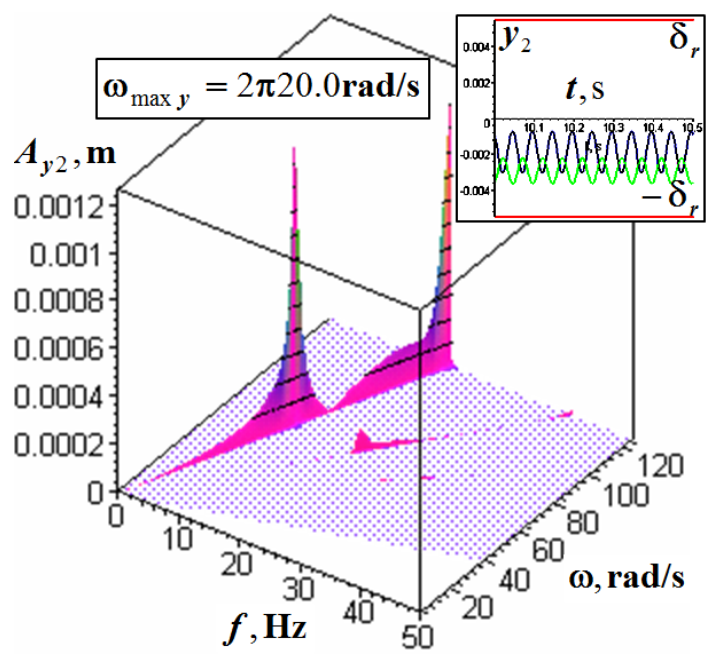

(d)

Figure 10. Results of order analysis of rotor motion vibrograms with FFT expansion into harmonics in the direction of generalised coordinates: (a) $x_{1}$, (b) $x_{2}$, (c) $y_{1}$, (d) $y_{2}$.

\section{System amplitude-frequency responses}

Figure 11 shows the first harmonic amplitudes $A_{1}$ vs. the field frequency dependence $\omega_{0}$ for generalised coordinates $y_{1}$ (light dotted line) and $y_{2}$ (dark solid line) under different 
CS operating conditions resulting from using the force characteristics of PMB and AMB: operating condition $I, F_{M 0}$ and $F_{M z 0}$, operating condition $I I, F_{M 2}$ (at $i_{b 1,2}=+3.5 \mathrm{~A}$ ) and $F_{M z 1}$, operating condition $I I I, F_{M 2}$ (at $i_{b 1,2}=-3.5 \mathrm{~A}$ ) and $F_{M z 2}$. This frequency was related to the rotor angular speed as $\omega_{0}=\omega / 2 \pi$. The graphs in Figure 11 show the dynamic behaviour of a rotor in the investigated range. Essentially, they were the projections of 3D spectra (Figure 10) onto coordinate planes $O \omega A$. The amplitude frequency responses corresponding to these three operating conditions are shown, respectively, in Figures 11(a-c). The horizontal lines show the geometrically possible deflections with the account of the static equilibrium position for each coordinate. The graphs also show the motion trajectories of the rotor radial support sections in the gap for three frequency values, viz. the $x$ resonance, the $y$ resonance and the range end. The AF responses shown demonstrate the possibility of transition through the first critical speed when the CS is operating in the designed condition $I$ without significant amplitude growth by smooth shock-free switching from one operating condition to another one, according to a predesigned program, e.g., I-III-II-I (Figure 11(d)).

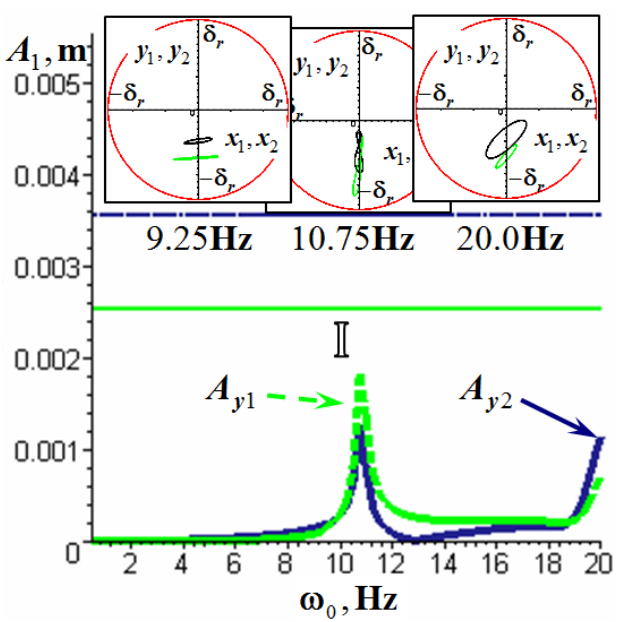

(a)

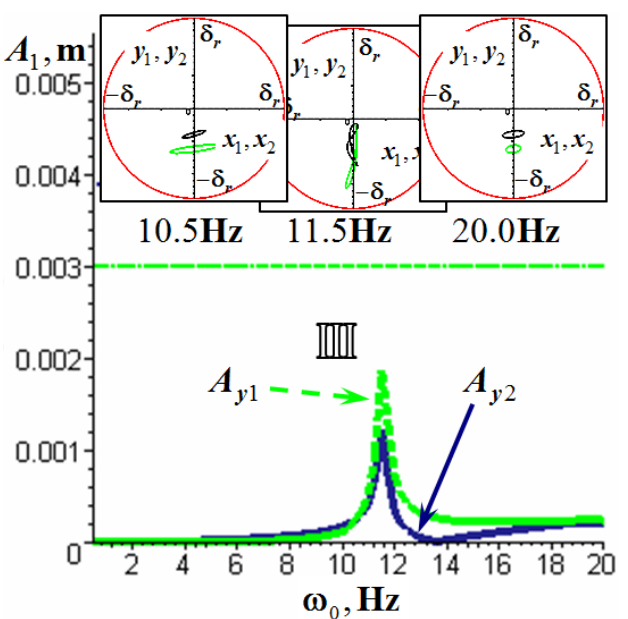

(c)

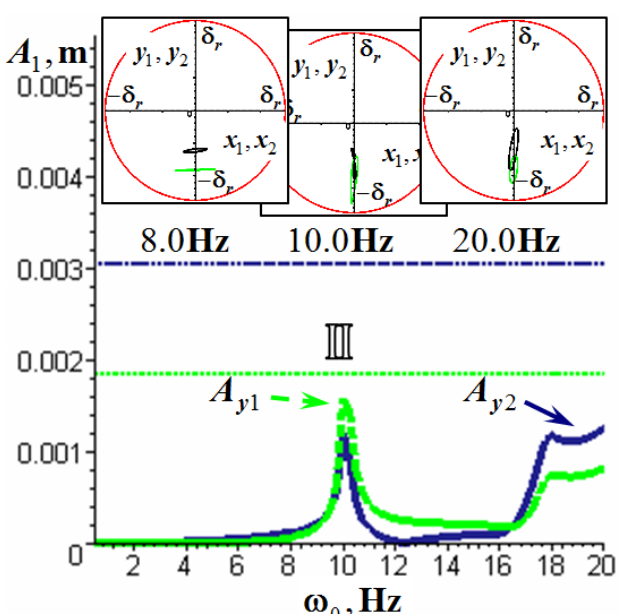

(b)

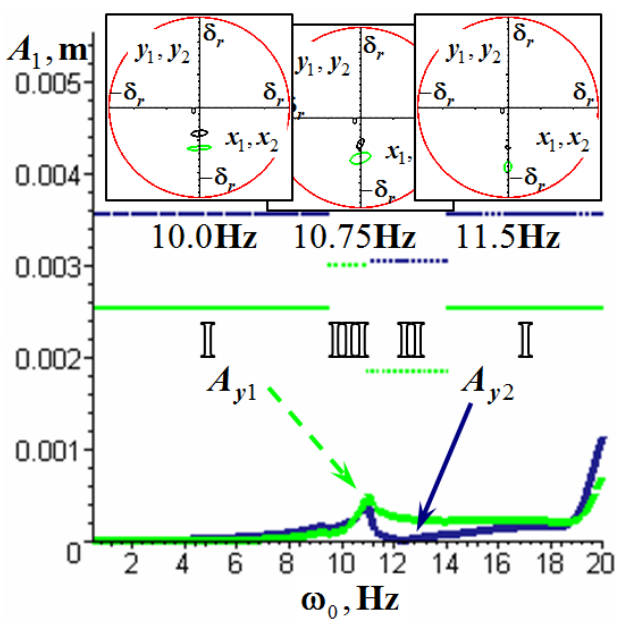

(d)

Figure 11. Amplitude-frequency responses for first harmonic at different CS operating conditions: (a) operating condition I; (b) operating condition II; (c) operating condition III;

(d) operating conditions I-III-II-I. 
Thus, Figure 11(d) illustrates the modelling of rotor passage through the first resonance without a substantial increase in vibration amplitudes. In the range of excitation frequencies $\omega_{0} 0$ to $9.5 \mathrm{~Hz}$ for PMB and AMB, the designed operating condition $I$ was activated. In this operating condition, resonance was observed at $10.75 \mathrm{~Hz}$ (Figure 11(a)). Then, during some time, a transition to operating condition III occurred. In this condition, the resonance occurred at $11.5 \mathrm{~Hz}$ (Figure $11(\mathrm{c})$ ), and at $10.75 \mathrm{~Hz}$ it was absent. After the rotor had sped up to $11 \mathrm{~Hz}$, transition to operating condition II occurred. This MB operating condition corresponded to a resonance at $10 \mathrm{~Hz}$ (Figure 11(b)), and at $11.5 \mathrm{~Hz}$ it was absent. Upon reaching $14 \mathrm{~Hz}$, a transition to design operating condition $I$ took place. This was how the amplitude of vibrations in the design operating condition (Figure 11(a)) was reduced by more than 4-fold (Figure 11(d)). Next, when the rotor had reached its operational speed, the process was repeated upon achieving each design operating condition I resonance. Each MB operating condition corresponded to its bearing stiffness that affected the resonance positions. Transition between operating conditions at each marginal frequency occurred during the pre-set time intervals. This ensured a smooth and bumpless transition. The study had shown that the analytical model used for computational research (in contrast to other, e.g., finite-element models) allows for the substantiated selection of practical and even optimal system parameters to implement the discussed method of detuning from critical modes. The model offered the advantage of performing a big number of trade-off studies to avoid a series of costly full-scale experiments and pre-commissioning activities.

\section{CONCLUSIONS}

Mathematical simulation and a series of experiments confirming its validity had proven that by selecting the parameters of the CS of the AMB or PMB with a bias winding, one can ensure such force characteristics of magnetic bearings, which will prevent rotor operation in a resonance mode and in the resonance zone of any critical speed within zero to working rotational speeds. The described method of supercritical rotor resonance in detuning and decreasing the amplitudes of resonance vibrations due to a short-term damping increase made AMB and PMB with a control winding a more preferable type of EDS as compared to other (mechanical) ones. The serviceability of a PMB with a control winding of the suggested design had been confirmed experimentally on a laboratory setup realising the combined passive-active magnetic suspension of a model rotor.

\section{ACKNOWLEDGEMENTS}

The research effort was conducted at the National Technical University "Kharkiv Polytechnic Institute" within the framework of R\&D activities funded by the state budget according to basic research programs of the Ministry of Education and Science of Ukraine (project no. 0115U000509). The author would like to be obliged to National Technical University "Kharkiv Polytechnic Institute" for providing laboratory facilities.

\section{REFERENCES}

[1] Genta G. Dynamics of rotating systems: Springer Science \& Business Media; 2007.

[2] Vance J, Zeidan F, Murphy B. Bearings and their effect on rotordynamics. Machinery vibration and rotordynamics. 2010:171-269. 
[3] Adams ML. Rotating machinery vibration: from analysis to troubleshooting: CRC Press; 2009.

[4] Schweitzer G. Applications and research topics for active magnetic bearings. IUTAM Symposium on Emerging Trends in Rotor Dynamics: Springer; 2011. p. 263-73.

[5] Bleuler H, Cole M, Keogh P, Larsonneur R, Maslen E, Okada Y, et al. Magnetic bearings: theory, design, and application to rotating machinery: Springer Science \& Business Media; 2009.

[6] Polajžer B. Magnetic bearings, theory and applications: Sciyo; 2010.

[7] Kamal M, Rahman MM. (2014). Finite element-based fatigue behaviour of springs in automobile suspension. International Journal of Automotive and Mechanical Engineering. 2014;10:1910-9.

[8] Jansen R, DiRusso E. Passive magnetic bearing with ferrofluid stabilization. 1996.

[9] Martynenko G. Magnetic bearings as the elastic-damping support rotors with controlled stiffness. . Bulletin of NTU "KhPI" Series: Dynamics and Strength of Machines Rusia. 2008. p. 111-24.

[10] Klimnjuk VJ EI. Method of safe acceleration of massive rotor of turbomachine installed in active magnetic bearings. Russia, June 2004.

[11] Ehskin ID KV. Method of Safe acceleration of massive vertical rotor of turbomachine installed in active magnetic bearings. Russia, March 2006.

[12] Bearing S. Magnetic Bearings for Combined Heat and Power Generation Plant. SKF Group PUB MT/S9: 15571 ENMarch 2015.

[13] Bearing S. SKF S2M Magnetic Bearings Control Cabinet E300V2. Advanced control for your oil and gas turbomachinery: SKF Group PUB BU/P2: 14966 EN; September 2014.

[14] Ibrahim TK, Rahman M, Mohammed M, Basrawi F. Statistical analysis and optimum performance of the gas turbine power plant International Journal of Automotive and Mechanical Engineering. 2016;13,1:3215-25.

[15] Ibrahim TK, Rahman M. Effects of isentropic efficiency and enhancing strategies on gas turbine performance. Journal of Mechanical Engineering and Sciences. 2013;4: 383-96.

[16] Simms J. Fundamentals of the Turboexpander: Basic Theory and Design. Simms Machinery International, Santa Maria, CA. 2009.

[17] Earnshaw S. On the nature of the molecular forces which regulate the constitution of the luminiferous ether. Transactions of the Cambridge Philosophical Society. 1842;7:97-112.

[18] Brounbeck W. Frein schweben diamagnetischen Körper in Magnetfeld. Physikalische Zeitschrift. 1939;112:764-9.

[19] Bassani R. Earnshaw (1805-1888) and passive magnetic levitation. Meccanica. 2006;41:375-89.

[20] Shukla S, Deheri G. Effect of slip velocity on magnetic fluid lubrication of rough porous rayleigh step bearing. Journal of Mechanical Engineering and Sciences. 2013;4:532-47.

[21] Martynenko G. The interrelated modelling method of the nonlinear dynamics of rigid rotors in passive and active magnetic bearings. Eastern-European Journal of Enterprise Technologies. 2016;2:4-13.

[22] Bekinal SI, Tumkur Ramakrishna AR, Jana S. Analysis of radial magnetized permanent magnet bearing characteristics. Progress In Electromagnetics Research B. 2013;47:87-105. 
[23] Azraai M, Priyandoko G, Yusoff A, Rashid M. Parametric Optimization of magneto-rheological fluid damper using particle swarm optimization. International Journal of Automotive and Mechanical Engineering. 2015;11:25919.

[24] Martynenko G. Application Of nonlinear models for a well-defined description of the dynamics of rotors in magnetic bearings. EUREKA: Physics and Engineering. 2016:3-12.

[25] Ehrich FF. Observations of nonlinear phenomena in rotordynamics. Journal of System Design and Dynamics. 2008;2:641-51.

[26] Martynenko G. Results of experimental research and analysis of the dynamics of a model rotor in magnetic bearings of different types. Bulletin of NTU "KhPI". Russia: Series: Dynamics and Strength of Machines; 2013. p. 90-101.

[27] Ji JC, Hansen C, Zander AC. Nonlinear dynamics of magnetic bearing systems. Journal of Intelligent Material Systems and Structures. 2008; 19(12): 1471-91.

[28] Lee JY. Sound and vibration signal analysis using improved short-time fourier representation. International Journal of Automotive and Mechanical Engineering. 2013;7:811-9. 